\title{
Spatiotemporal vortex beams and angular momentum
}

\author{
Konstantin Y. Bliokh ${ }^{1,2}$ and Franco Nori ${ }^{1,3}$ \\ ${ }^{1}$ Advanced Science Institute, RIKEN, Wako-shi, Saitama 351-0198, Japan \\ ${ }^{2}$ A. Usikov Institute of Radiophysics and Electronics, NASU, Kharkov 61085, Ukraine \\ ${ }^{3}$ Physics Department, University of Michigan, Ann Arbor, Michigan 48109-1040, USA
}

(Received 16 June 2012; published 19 September 2012)

\begin{abstract}
We present a space-time generalization of the known spatial (monochromatic) wave vortex beams carrying intrinsic orbital angular momentum (OAM) along the propagation direction. Generic spatiotemporal vortex beams are polychromatic and can carry intrinsic OAM at an arbitrary angle to the mean momentum. Applying either (i) a transverse wave-vector shift or (ii) a Lorentz boost to a monochromatic Bessel beam, we construct a family of either (i) time-diffracting or (ii) nondiffracting spatiotemporal Bessel beams, which are exact solutions of the Klein-Gordon wave equations. The proposed spatiotemporal OAM states are able to describe either photon or electron vortex states (both relativistic and nonrelativistic) and have potential applications in particle collisions, optics of moving media, quantum communications, and astrophysics.
\end{abstract}

DOI: 10.1103/PhysRevA.86.033824

PACS number(s): 42.50.Tx, 42.65.Sf

\section{INTRODUCTION}

Wave-front dislocations (phase singularities or optical vortices) were introduced in 1974 in a seminal paper by Nye and Berry [1] which gave rise to the field of singular optics [2]. A nonsingular wave locally looks like a segment of a plane wave with parallel wave fronts, a well-defined phase, and current flowing in the direction of wave propagation. Nye and Berry [1] demonstrated that, in general, the wave fronts in three-dimensional space contain topological dislocation lines, akin to those in crystal lattices, where the phase becomes singular and currents coil around the lines forming optical vortices. The dislocations are characterized by a quantized vortex strength, also called topological charge. One can distinguish screw and edge wave-front dislocations where the vortex lines are, respectively, parallel and orthogonal to the wave propagation direction. In general, mixed edgescrew dislocations, with the vortex core tilted with respect to the phase fronts, are possible. Importantly, only screw dislocations are generic in monochromatic wave fields, while generic edge-screw dislocations require additional temporal variations [1,2].

In 1992 Allen et al. [3] revealed a close relation between optical vortices and wave orbital angular momentum (OAM). They showed that axially symmetric higher-order optical beams in free space bear screw dislocations along their axes and possess intrinsic OAM directed along the beam axis. This OAM is quantized with its magnitude (in units of $\hbar$ per one photon) being equal to the vortex charge. Thus, monochromatic vortex beams represent the OAM states of light, and nowadays they play an important role in classical and quantum optics [4]. Remarkably, the above phase-dislocation, vortex, and OAM properties are generic for all types of linear waves, both classical and quantum, independently of their nature. In particular, vortex beams with OAM were recently described and generated in electron microscopes [5,6], as well as employed in acoustics [7].

For monochromatic vortex wave beams, the intrinsic OAM is collinear to the momentum, with its projection on the beam axis (helicity) being quantized. In other words, such OAM behaves very similar to the spin of a massless particle (even for nonrelativistic electrons [5]). Then, a natural question arises: Can a generic wave packet or beam carry a well-defined intrinsic OAM in an arbitrary direction, i.e., tilted with respect to the propagation direction? It would seem that the direction of the intrinsic OAM is associated with the direction of the vortex line, and the tilted OAM should appear in states with tilted vortices, i.e., mixed edge-screw wave-front dislocations. As we pointed out, such dislocations are generic for polychromatic fields and our question essentially requires studying vortex beams and OAM in space-time $[8,9]$.

In this paper, we address the above question and extend the concepts of intrinsic OAM and vortex beams to polychromatic states in space-time. We analyze the scalar Klein-Gordon wave equation, so that its relativistic and nonrelativistic limits describe both massless optical fields and massive Schrödinger particles, assuming that the polarization effects can be neglected. We show that the existence of spatiotemporal vortex beams with tilted OAM follows from the requirement of relativistic invariance and can be obtained via Lorentz transformations of the usual spatial vortex beams [9]. Hence, the spatiotemporal OAM wave states can naturally appear from moving sources emitting stationary (monochromatic) vortex states in their rest frames. As such, our results have implications in the optics of moving media [10], quantum communications with satellites [11], collisions of high-energy particles with OAM [12], and astrophysical applications of OAM of light $[13,14]$.

\section{MONOCHROMATIC BESSEL BEAMS}

We start with the Klein-Gordon wave equation in units $\hbar=c=1$ :

$$
\left(-\partial_{t}^{2}+\nabla^{2}-m^{2}\right) \psi=0
$$

where $\psi(t, \mathbf{r})$ is the scalar wave function. The plane-wave solutions of this equation are

$$
\begin{aligned}
& \psi \propto \exp [i(-\omega t+\mathbf{k} \cdot \mathbf{r})] \equiv \exp \left(i k^{\mu} r_{\mu}\right), \\
& \omega^{2}-k^{2} \equiv k^{\mu} k_{\mu}=m^{2},
\end{aligned}
$$


with $k^{\mu}=(\omega, \mathbf{k})$ and $r^{\mu}=(t, \mathbf{r})$ being the standard fourvectors in the Minkowski space-time with signature $(-,+$, $+,+)$. The second equality in Eq. (2) is the dispersion relation which determines the mass hyperboloid (or the light cone at $m=0$ ) - a hypersurface in momentum $k^{\mu}$-space, where we only consider the positive-energy domain $\omega>0$. We deal with the Klein-Gordon equation because it possesses relativistic space-time symmetry and is able to describe both quantum massive particles of different energies and classical waves. (Indeed, for $k \ll m$ it can be reduced to the Schrödinger equation, whereas in the relativistic limit $k \gg m$ it becomes the usual wave equation.) Alongside the wave function $\psi(t, \mathbf{r})$, we use its plane-wave (Fourier) spectrum $\tilde{\psi}(\omega, \mathbf{k})$, defined with the explicit $\delta$ function of the dispersion (2):

$$
\psi\left(r^{\mu}\right) \propto \int \tilde{\psi}\left(k^{\mu}\right) \delta\left(k^{\mu} k_{\mu}-m^{2}\right) e^{i k^{\mu} r_{\mu}} d^{4} k^{\mu} .
$$

The simplest monochromatic solutions of wave equations, which carry OAM, are Bessel beams [15]. These represent eigenmodes of the $z$ components of the momentum, $\hat{\mathbf{p}}=-i \nabla$, and the OAM, $\hat{\mathbf{L}}=\hat{\mathbf{r}} \times \hat{\mathbf{p}}$, and can be constructed as a superposition of multiple plane waves (2) with fixed $\omega=\omega_{0}$ and $k_{z}=k_{z 0}$. The fixed frequency implies that the wave vectors form an isofrequency sphere $k=\sqrt{\omega_{0}^{2}-m^{2}} \equiv k_{0}$ in $\mathbf{k}$ space, whereas a fixed $k_{z}$ cuts a circle $k_{\perp}=\sqrt{\omega_{0}^{2}-m^{2}-k_{z 0}^{2}} \equiv k_{\perp 0}$ on this sphere see [Fig. 1(a)]. Hereafter $\left(k_{\perp}, \phi, k_{z}\right)$ denote the cylindrical coordinates in $\mathbf{k}$ space. The relative phases of plane waves can grow around the circle and form a vortex $\exp (i \ell \phi)$ with phase increment $2 \pi \ell$ around the loop, whereas the vortex charge $\ell=0, \pm 1, \pm 2, \ldots$ determines the order of the Bessel beam and its OAM. Thus, the plane-wave spectrum of the $\ell$ th-order beam can be written as

$$
\tilde{\psi}_{\ell}(\omega, \mathbf{k}) \propto \delta\left(k-k_{0}\right) \delta\left(k_{\perp}-k_{\perp 0}\right) \exp (i \ell \phi) .
$$

The corresponding real-space field (3) yields

$$
\psi_{\ell}(t, \mathbf{r}) \propto J_{|\ell|}\left(k_{\perp 0} r_{\perp}\right) \exp \left[i\left(\ell \varphi+k_{z 0} z-\omega_{0} t\right)\right],
$$

where $J_{n}(\xi)$ is the Bessel function of the first kind and $\left(r_{\perp}, \varphi, z\right)$ are cylindrical coordinates in real space. In the vicinity of the beam axis, $k_{\perp 0} r_{\perp} \ll 1$,

$$
\psi_{\ell} \propto[x+i \operatorname{sgn}(\ell) y]^{|\ell|} \exp \left[i\left(k_{z 0} z-\omega_{0} t\right)\right]
$$

which demonstrates a screw wave-front dislocation of strength $\ell$ on the axis [1,2].

Bessel beams (5) are nondiffracting; i.e., $\left|\psi_{\ell}\right|^{2}$ does not vary with $z$ and $t$ [15]. Evidently, these are eigenmodes of the operators $\hat{p}_{z}=-i \partial_{z}$ and $\hat{L}_{z}=-i \partial_{\varphi}$, with corresponding eigenvalues $k_{z}$ and $\ell$. Figure 1(b) shows the transverse spatial distributions of the intensity (probability density) $\rho=|\psi|^{2}$, the current (momentum density) $\mathbf{j}=\operatorname{Im}\left(\psi^{*} \nabla \psi\right)=\rho \nabla \Phi$, and the phase $\Phi=\arg \psi$ for the beam (5). It is the screw phase dislocation at $r_{\perp}=0$ and the accompanying vortex current that produce a nonzero OAM of the field.

To characterize the angular momentum, it is important to also describe the expectation values of energy-momentum and coordinates of the beam $[9,16]$. The normalized (per one particle) expectation values of the energy, transverse
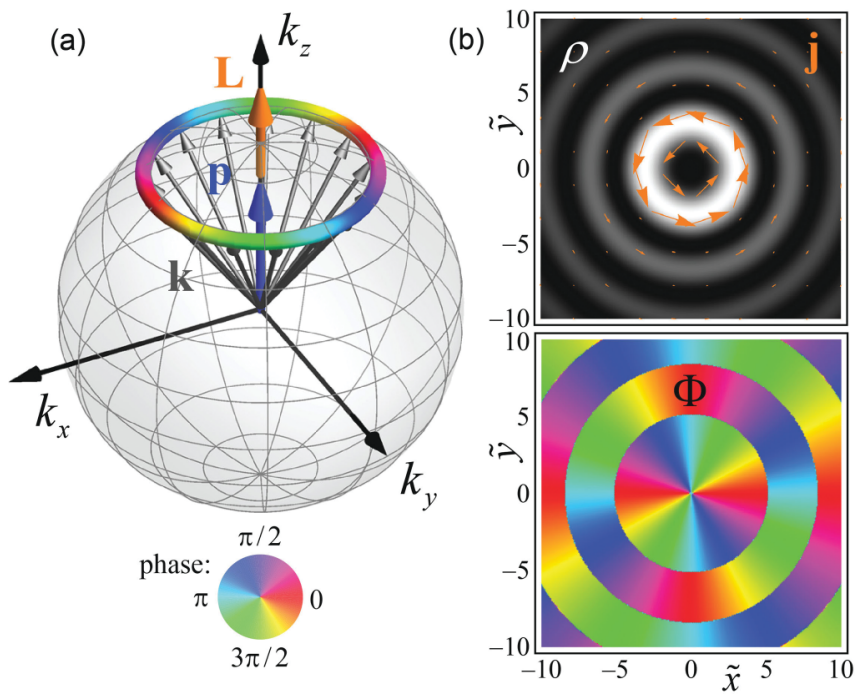

FIG. 1. (Color online) (a) Plane-wave spectrum (4) of a monochromatic Bessel beam with $\ell=2$ : a circle on the isofrequency sphere $k=\sqrt{\omega_{0}^{2}-m^{2}}$ in $\mathbf{k}$ space. Relative phases of the constituent waves form a charge- 2 optical vortex encoded by colors. (b) Transverse real-space distributions of the intensity $\rho=|\psi|^{2}$, the current $\mathbf{j}=\operatorname{Im}\left(\psi^{*} \nabla \psi\right)=\rho \nabla \Phi$, and the phase $\Phi=\arg \psi$ for the Bessel beam (5) corresponding to panel (a). The phase singularity on the beam axis represents a screw wave-front dislocation accompanied by a vortex of the current. The parameters used here are $\omega_{0} / m=2$, $k_{\perp 0} / m=1$, and $k_{z 0} / m=\sqrt{2}$, and the dimensionless coordinates are $\tilde{x}=k_{\perp 0} x$ and $\tilde{y}=k_{\perp 0} y$. The normalized momentum and the OAM, Eqs. (6) and (7), integrated over the visible area, yield $\mathbf{p}=k_{z 0} \mathbf{e}_{z}$ and $\mathbf{L}=\ell \mathbf{e}_{z}$.

coordinates, momentum, and OAM for the Bessel beam are

$$
\begin{aligned}
& E=\frac{\left\langle\psi\left|i \partial_{t}\right| \psi\right\rangle}{\langle\psi \mid \psi\rangle}=\omega_{0}, \quad \mathbf{R}_{\perp}=\frac{\left\langle\psi\left|\mathbf{r}_{\perp}\right| \psi\right\rangle}{\langle\psi \mid \psi\rangle}=\mathbf{0}, \\
& \mathbf{p}=\frac{\langle\psi|-i \nabla| \psi\rangle}{\langle\psi \mid \psi\rangle}=k_{z 0} \mathbf{e}_{z}, \quad \mathbf{L}=\frac{\langle\psi|\hat{\mathbf{L}}| \psi\rangle}{\langle\psi \mid \psi\rangle}=\ell \mathbf{e}_{z} .
\end{aligned}
$$

Hereafter $\mathbf{e}_{a}$ denotes the unit basis vector of the corresponding $a$ axis, whereas the subscript " $\perp$ " indicates the transverse $(x, y)$ components of a vector. As expected, the OAM is collinear with the momentum, $\mathbf{L} \| \mathbf{p}$. The inner product in Eq. (6) implies integration over the proper area of space. Formally, the Bessel beams are not localized, i.e., cannot be normalized in the whole space. However, this can be overcome by substituting $\delta$ functions in the spectrum (4) with arbitrarily narrow Gaussian exponents, and the normalized expectation values (6) converge to finite values. An alternative form of Eqs. (6) using the probability density $\rho$ and momentum density j distributions can be written as [17]

$$
\begin{aligned}
\mathbf{R}_{\perp} & =\frac{\int \mathbf{r}_{\perp} \rho d^{2} \mathbf{r}_{\perp}}{\int \rho d^{2} \mathbf{r}_{\perp}}=\mathbf{0}, \quad \mathbf{p}=\frac{\int \mathbf{j} d^{2} \mathbf{r}_{\perp}}{\int \rho d^{2} \mathbf{r}_{\perp}}=k_{z 0} \mathbf{e}_{z}, \\
\mathbf{L} & =\frac{\int(\mathbf{r} \times \mathbf{j}) d^{2} \mathbf{r}_{\perp}}{\int \rho d^{2} \mathbf{r}_{\perp}}=\ell \mathbf{e}_{z} .
\end{aligned}
$$

This makes it clear that it is the circulation of the current $\mathbf{j}$ [shown in Fig. 1(b)] that produces the intrinsic OAM of the beam. Note that the proper probability density and momentum 

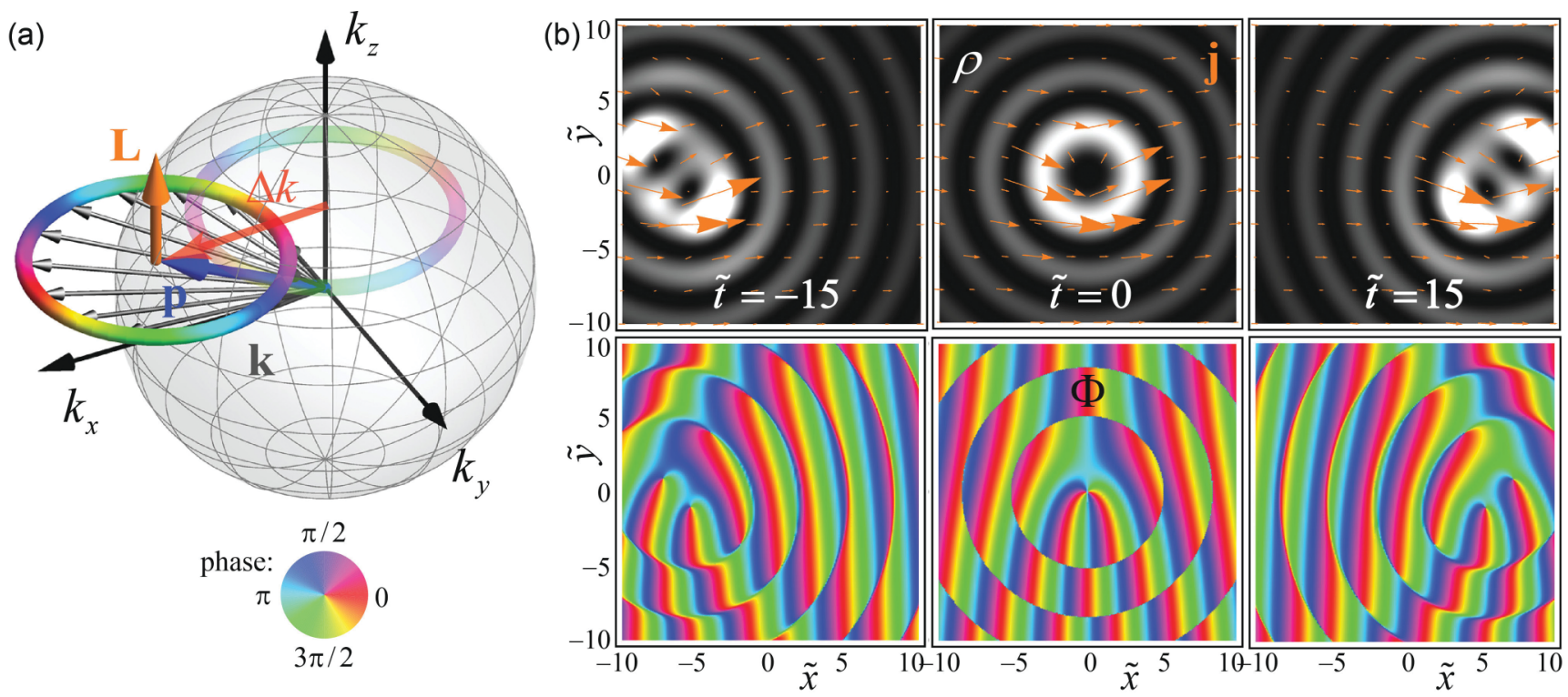

FIG. 2. (Color online) (a) Plane-wave spectrum of a polychromatic Bessel beam with $\ell=2$ and spectrum (9) shifted by $\Delta k \mathbf{e}_{x}$ in $\mathbf{k}$ space. Here the semitransparent circle represents the initial monochromatic beam, and the isofrequency sphere has radius $\sqrt{k_{z 0}^{2}+\Delta k^{2}}$. (b) Transverse real-space distributions of the intensity $\rho$, the current $\mathbf{j}$, and the phase $\Phi$ for the beam (a) at different times $t$. The beam moves in the positive $x$ direction and experiences time-diffraction deformations. The forklike phase patterns in the beam center represent moving edge-screw wave-front dislocations. The parameters are $k_{z 0} / m=1, k_{\perp 0} / m=1.4, \Delta k / m=2$, and $\omega_{c} / m=2.45$, and we use the dimensionless variables $\tilde{x}=k_{\perp 0} x, \tilde{y}=k_{\perp 0} y$, and $\tilde{t}=\omega_{c} t$. The normalized expectation values, Eqs. (6) and (7), numerically integrated over the same area around the moving beam center yield Eqs. (11) with a good accuracy: $\mathbf{R}_{\perp} \simeq\left(\Delta k / \omega_{c}\right) t \mathbf{e}_{x}, \mathbf{p} \simeq k_{z 0} \mathbf{e}_{z}+\Delta k \mathbf{e}_{x}$, and $\mathbf{L} \simeq \ell \mathbf{e}_{z} \nVdash \mathbf{p}$.

density for the relativistic Klein-Gordon equation differ from the "naive" $\rho$ and $\mathbf{j}$ used here $[9,18]$. However, this does not affect our consideration and is discussed below in Sec. V.

In practice, the Bessel-beam spectrum (4) can be simplified and well approximated by a finite number $N \gg 1$ of plane waves distributed over the spectral circle in $\mathbf{k}$ space [see Fig. 1(a)] [14]. In this manner,

$$
\begin{aligned}
\psi_{\ell}(t, \mathbf{r}) & \propto \frac{1}{\sqrt{N}} \sum_{n=1}^{N} \exp \left[i\left(-\omega_{n} t+\mathbf{k}_{n} \cdot \mathbf{r}+\ell \phi_{n}\right)\right], \\
\omega_{n} & =\omega_{0}, \quad \mathbf{k}_{n}=\left(k_{\perp 0} \cos \phi_{n}, k_{\perp 0} \sin \phi_{n}, k_{z 0}\right), \\
\phi_{n} & =\frac{2 \pi}{N} n .
\end{aligned}
$$

The discretization (8) is important for interferometric applications [14] and approximates well the beams (5) in the restricted area around the axis: e.g., $k_{\perp 0} r_{\perp} \leqslant 10$ for $N=20$. In Fig. 1 and throughout this paper we use the superposition (8) of $N=$ 30 plane waves for numerical simulations of Bessel beams, because in some of the cases described below the beams cannot be characterized analytically. We verified numerically that the normalized energy, momentum, and OAM of such beams are in perfect agreement with Eqs. (6) and (7) when the integration is performed over the area $S=\left\{\left|k_{\perp 0} x\right|<10,\left|k_{\perp 0} y\right|<10\right\}$.

\section{SPATIOTEMPORAL BESSEL BEAMS WITH SHIFTED SPECTRUM}

From the above picture of the monochromatic Bessel beams, one can see that the expectation values of the OAM and the momentum are collinear not by chance. Indeed, assuming a circular plane-wave distribution in momentum space [Fig. 1(a)], geometrically, $\mathbf{p}$ represents the radius vector of the center of the circle, whereas $\mathbf{L}$ points in the direction normal to the circle and has a magnitude equal to the vortex charge $\ell$. Obviously, the collinearity $\mathbf{L} \| \mathbf{p}$ holds true for any circle on the isofrequency sphere, i.e., for any axially symmetric monochromatic beam. However, as soon as we abandon the monochromaticity constraint, it is possible to construct a Bessel-beam-type solution with $\mathbf{L} \nVdash \mathbf{p}$. Indeed, any circle in $\mathbf{k}$ space can serve as a plane-wave spectrum for the beam. The only fundamental constraint of the mass hyperboloid (2) can always be satisfied by choosing the corresponding frequencies $\omega(\mathbf{k})=\sqrt{m^{2}+k^{2}}$. Thus, considering different spectral circles with vortex phases [see an example in Fig. 2(a)], we obtain different vortex solutions of the wave equation (1). It is easy to show that the center of the circle and the normal to the circle still represent the mean momentum and intrinsic OAM of the beam, respectively.

The simplest transformation shifting the spectral circle away from the isofrequency sphere is a uniform transverse shift (e.g., along the $x$ axis) of all the wave vectors in the Bessel-beam spectrum [Fig. 2(a)]:

$$
\mathbf{k} \rightarrow \mathbf{k}+\Delta k \mathbf{e}_{x}, \quad \omega_{0} \rightarrow \omega(\mathbf{k})=\sqrt{\omega_{0}^{2}+\Delta k^{2}+2 k_{x} \Delta k} .
$$

Solutions with spectrum (9) are polychromatic because of the $\omega\left(k_{x}\right)$ dependence (here $k_{x}$ is the wave-vector component of the original monochromatic beam). Remarkably, the beam spectrum (9) represents not only a circle with an azimuthal phase gradient when projected onto the $\left(k_{x}, k_{y}\right)$ plane but also a similar vortex loop around $\omega=\omega_{c} \equiv \sqrt{\omega_{0}^{2}+\Delta k^{2}}$ 
(a)

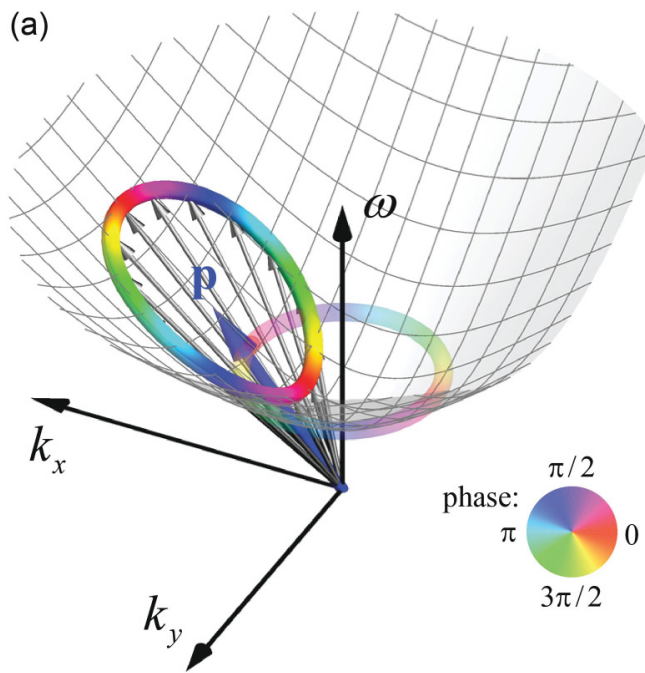

(b) 10

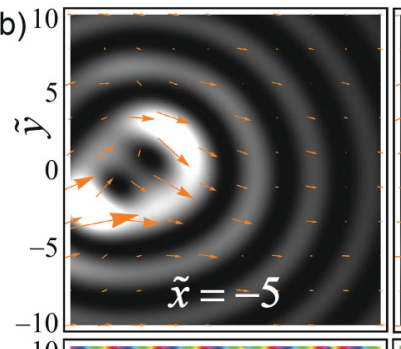

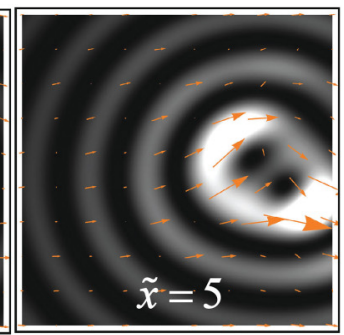

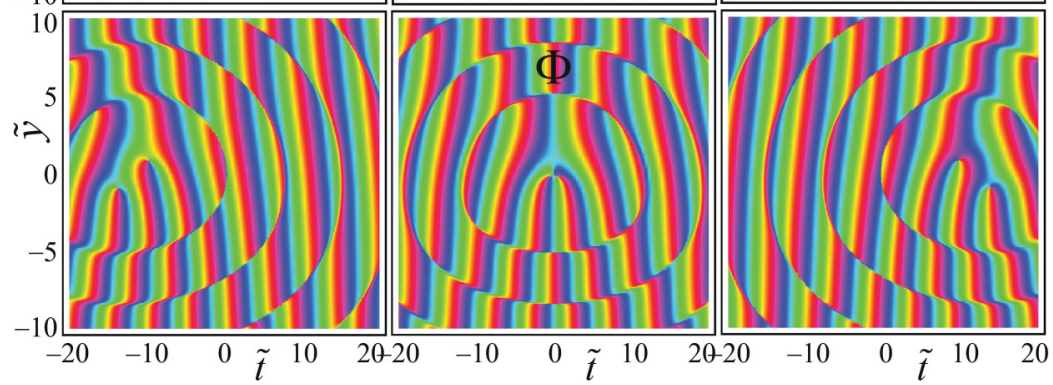

FIG. 3. (Color online) Spatiotemporal representation of the transversely moving Bessel beam from Fig. 2. (a) Here its spectrum is plotted on the mass hyperboloid in the $\left(\omega, k_{x}, k_{y}\right)$ space where it is seen that it also forms a vortex loop if projected onto the $\left(\omega, k_{y}\right)$ plane. (b) Real-space evolution shown here in the form of the distributions in the $(t, y)$ plane, for different values of $x$ [cf. Fig. 2(b)]. The corresponding components of the four-current $j^{\mu}$ are $\left(j^{0}, j_{y}\right)$, where $j^{0}=-\operatorname{Im}\left(\psi^{*} \partial_{t} \psi\right)$.

in the $\left(\omega, k_{y}\right)$ plane. Thus, this is a vortex loop on the mass hyperboloid in $(\omega, \mathbf{k})$ space, which demonstrates its spatiotemporal nature [see Fig. 3(a)].

Formally, the wave function of the beam can be written as a Fourier integral (3) with the $\delta$-function spectrum (4) shifted by the transformation (9). However, such an integral cannot be evaluated analytically in the general case. To understand properties of the beams with a shifted spectrum, we performed numerical simulations using the discretization (8) with parameters corresponding to Eq. (9):

$$
\begin{aligned}
& \omega_{n}=\sqrt{\omega_{0}^{2}+\Delta k^{2}+2 k_{\perp 0} \Delta k \cos \phi_{n}}, \\
& \mathbf{k}_{n}=\left(\Delta k+k_{\perp 0} \cos \phi_{n}, k_{\perp 0} \sin \phi_{n}, k_{z 0}\right) .
\end{aligned}
$$

The results are presented in Fig. 2(b). We see a Bessel-beam solution which is still homogeneous in the $z$ direction $\left(k_{z}=\right.$ $\left.k_{z 0}\right)$ but evolving in time. At $t=0$, the intensity distribution represents the Bessel function, $\rho(\mathbf{r}, t=0) \propto\left[J_{|\ell|}\left(k_{\perp 0} r_{\perp}\right)\right]^{2}$, but the current and phase distributions differ significantly as compared with the monochromatic beam (5) [Fig. 1(b)]. Indeed, there is a net transverse current flowing in the $x$ direction (because of the mean $\left\langle k_{x}\right\rangle=\Delta k$ ), whereas the phase dislocation in the beam center becomes of the mixed edge-screw type. This is seen from the forklike pattern of the phase fronts instead of the radial pattern characteristic of screw dislocations. Calculating the wave-field distributions at $t \neq 0$, we see that the beam moves in the transverse $x$ direction and experiences shape distortions. The deformations represent diffraction in time caused by the accumulated phase difference between waves with different frequencies (10). In particular, Fig. 2(b) shows that this diffraction breaks the $\ell$ th-order phase dislocation at $t=0$ into $|\ell|$ basic dislocations of strengths at $t \neq 0$. This can be regarded as a temporal manifestation of the general instability of higher-order vortices with respect to perturbations $[1,2,19]$.
The moving edge-screw dislocation reveals a spatiotemporal character of the shifted-spectrum vortex beam. To illustrate this, we plot the beam density, current, and phase distributions not only in the $(x, y)$ plane for different values of $t$ [Fig. 2(b)] but also in the $(t, y)$ plane for different values of $x$ [Fig. 3(b)]. One can see quite similar "moving" beams with edge-screw phase dislocation and circulating probability current in both the $(x, y)$ and $(t, y)$ planes. Thus, in general, this is a vortex "hyperbeam" in Minkowski $(t, \mathbf{r})$ space-time.

The transverse motion of the beam implies a nonzero transverse momentum, $p_{x} \neq 0$, whereas the OAM is still determined by the $z$ direction of the vortex axis (which also moves along $x$ with time). Calculating the expectation values (6) in the momentum (Fourier) representation and assuming paraxiality of the beam, $k_{\perp 0} \ll \sqrt{k_{z 0}^{2}+\Delta k^{2}}$, we obtain

$$
\begin{aligned}
& E \simeq \omega_{c}=\sqrt{\omega_{0}^{2}+\Delta k^{2}}, \quad \mathbf{R}_{\perp} \simeq \frac{\Delta k}{\omega_{c}} t \mathbf{e}_{x}, \\
& \mathbf{p} \simeq k_{z 0} \mathbf{e}_{z}+\Delta k \mathbf{e}_{x}, \quad \mathbf{L} \simeq \ell \mathbf{e}_{z} .
\end{aligned}
$$

Here the beam centroid moves in the transverse direction according to the free-space equation of motion: $d \mathbf{R}_{\perp} / d t=$ $\mathbf{p}_{\perp} / E$. Numerical calculations of the expectation values in the representation (7), performed using the same area around the instantaneous transverse beam centroid $\mathbf{R}_{\perp}$, showed good agreement with Eq. (11) even for the nonparaxial parameters used in Fig. 2. Thus, Figs. 2 and 3, together with Eqs. (11), demonstrate the existence of spatiotemporal vortex beams with noncollinear momentum and intrinsic OAM: L $¥ \mathbf{p}$.

\section{LORENTZ-BOOSTED SPATIOTEMPORAL BESSEL BEAMS}

It turns out that it is possible to construct a family of spatiotemporal beams which are free of the temporal diffraction and allow a simple analytic description. Note that Lorentz 

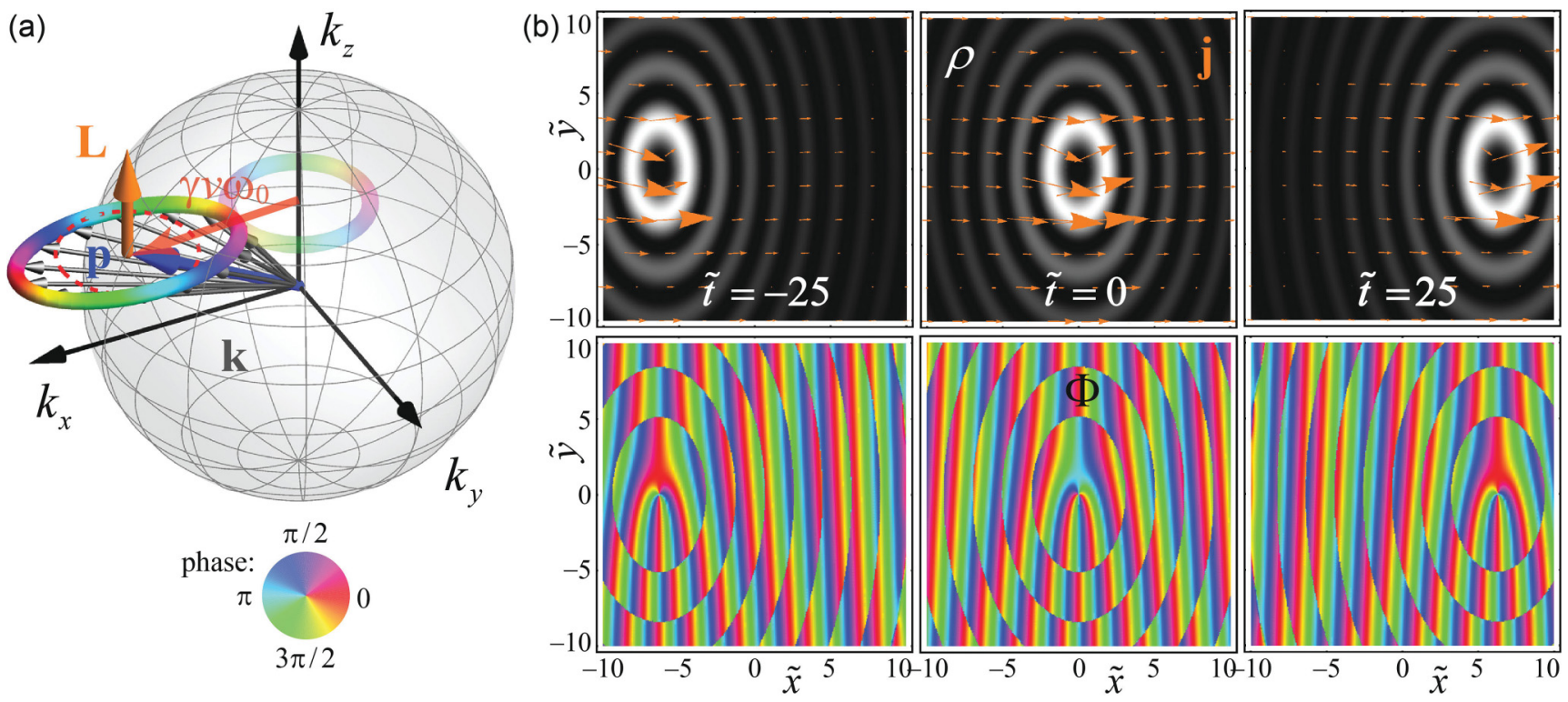

FIG. 4. (Color online) (a) Plane-wave spectrum (15) of a Lorentz-boosted Bessel beam with $\ell=2$ in $\mathbf{k}$ space. Here the semitransparent circle represents the initial monochromatic beam, whereas the isofrequency sphere has radius $\sqrt{k_{z 0}^{2}+\left(\gamma v \omega_{0}\right)^{2}}$. (b) Transverse real-space distributions of the probability density $\rho$, the current $\mathbf{j}$, and the phase $\Phi$ for the beam (14) corresponding to panel (a), at different times $t$. The beam moves uniformly in the positive $x$ direction without diffraction. The forklike phase pattern represents a moving edge-screw wave-front dislocation. The parameters are $\omega_{0} / m=2, \kappa_{0} / m=1, \Delta k / m=2$, and $v=0.8(\gamma=5 / 3)$, and we use the dimensionless variables $\tilde{x}=k_{\perp 0} x$, $\tilde{y}=k_{\perp 0} y$, and $\tilde{t}=\omega_{c} t$. The numerically calculated normalized expectation values, Eqs. (6) and (7), correspond to Eq. (18) with good accuracy.

transformation to a moving reference frame, $r^{\mu} \rightarrow \hat{\Lambda}_{v}^{\mu}(\mathbf{v}) r^{v}$ ( $\mathbf{v}$ is velocity), provides a boost of the four-momentum in the spectrum, $k^{\mu} \rightarrow \hat{\Lambda}_{v}^{\mu}(-\mathbf{v}) k^{\nu}$, and can generate polychromatic moving solutions. Since the Klein-Gordon wave equation is Lorentz invariant, the Lorentz-boosted Bessel beams (4) and (5) also represent exact solutions of Eq. (1):

$$
\psi_{\ell}^{\prime}\left(r^{\nu}\right) \equiv \psi_{\ell}\left[\hat{\Lambda}_{\nu}^{\mu}(\mathbf{v}) r^{\nu}\right] \text { and } \tilde{\psi}_{\ell}^{\prime}\left(k^{\nu}\right) \equiv \tilde{\psi}_{\ell}\left[\hat{\Lambda}_{v}^{\mu}(-\mathbf{v}) k^{\nu}\right] .
$$

Equations (12), with Eqs. (4) and (5), offer a new family of moving vortex beams parametrized by the velocity $\mathbf{v}$.

It is easy to see that the Lorentz boosts in the longitudinal $z$ direction keep the monochromatic Bessel-beam family, Eqs. (4) and (5), invariant and only transform their parameters $\omega_{0}, k_{z 0}$, and $k_{\perp 0}$. Therefore, we consider only the nontrivial case of the transverse boost, say, in the $x$ direction. Assuming $\mathbf{v}=v \mathbf{e}_{x}$, we have

$$
\hat{\Lambda}_{v}^{\mu}(\mathbf{v})=\left(\begin{array}{cccc}
\gamma & -v \gamma & 0 & 0 \\
-v \gamma & \gamma & 0 & 0 \\
0 & 0 & 1 & 0 \\
0 & 0 & 0 & 1
\end{array}\right),
$$

with $\gamma=1 / \sqrt{1-v^{2}}$ being the Lorentz factor. Explicitly, the moving Bessel beam (12) with Eq. (13) is obtained via the substitution $t \rightarrow \gamma(t-v x)$ and $x \rightarrow \gamma(x-v t)$ in Eq. (5), and it takes the form

$$
\begin{aligned}
& \psi_{\ell}^{\prime}(t, \mathbf{r}) \propto J_{|\ell|}\left(k_{\perp 0} r_{\perp}^{\prime}\right) \exp \left[i\left(\ell \varphi^{\prime}+k_{z 0} z-\omega_{0} t^{\prime}\right)\right], \\
& r_{\perp}^{\prime}=\sqrt{\gamma^{2}(x-v t)^{2}+y^{2}}, \quad \varphi^{\prime}=\tan ^{-1}\left(\gamma \frac{x-v t}{y}\right), \\
& t^{\prime}=\gamma(t-v x) .
\end{aligned}
$$

At the same time, the Lorentz transformation, Eqs. (12) and (13), of the spectral characteristics yields [cf. Eq. (9)]

$$
k_{x} \rightarrow \gamma\left(k_{x}+v \omega_{0}\right) \text { and } \omega_{0} \rightarrow \omega(\mathbf{k})=\gamma\left(\omega_{0}+v k_{x}\right) .
$$

In $\mathbf{k}$ space, this represents a shift of the spectral circle by the distance $\Delta k_{x}=\gamma v \omega_{0}$ and a stretch with factor $\gamma$ along the $k_{x}$ dimension [see Fig. 4(a)]. This stretching of the spectrum ensures the linearity of the $\omega\left(k_{x}\right)$ dependence which causes no diffraction in time. Plotting the Lorentz-boosted spectrum $(15)$ in the $(\omega, \mathbf{k})$ space, one can see an elliptic planar loop on the mass hyperboloid [Fig. 5(a)]. One can consider this as a vortex loop both when projected onto the $\left(k_{x}, k_{y}\right)$ plane and also when projected onto the $\left(\omega, k_{y}\right)$ plane, which demonstrates the spatiotemporal character of this vortex. The spectral discretization (8) for the Lorentz-boosted beam acquires the following form according to Eq. (15):

$$
\begin{aligned}
& \omega_{n}=\gamma\left(\omega_{0}+v k_{\perp 0} \cos \phi_{n}\right), \\
& \mathbf{k}_{n}=\left(\gamma\left(k_{\perp 0} \cos \phi_{n}+v \omega_{0}\right), k_{\perp 0} \sin \phi_{n}, k_{z 0}\right) .
\end{aligned}
$$

Figure 4(b) shows the real-space distributions of the intensity, the current, and the phase in the Lorentz-boosted Bessel beam (14). This beam moves in the transverse $x$ direction with velocity $\mathbf{v}=v \mathbf{e}_{x}$ and has an elliptical profile (stretching of $k_{x}$ yields the Lorentz contraction of the $x$ dimension with factor $\gamma$ ). The wave function (14) near the beam axis, $k_{\perp 0} r_{\perp}^{\prime} \ll 1$, is approximated by

$$
\psi_{\ell}^{\prime} \propto[\gamma(x-v t)+i \operatorname{sgn}(\ell) y]^{|\ell|} \exp \left\{i\left[k_{z 0} z+\omega_{0} \gamma(v x-t)\right]\right\} .
$$



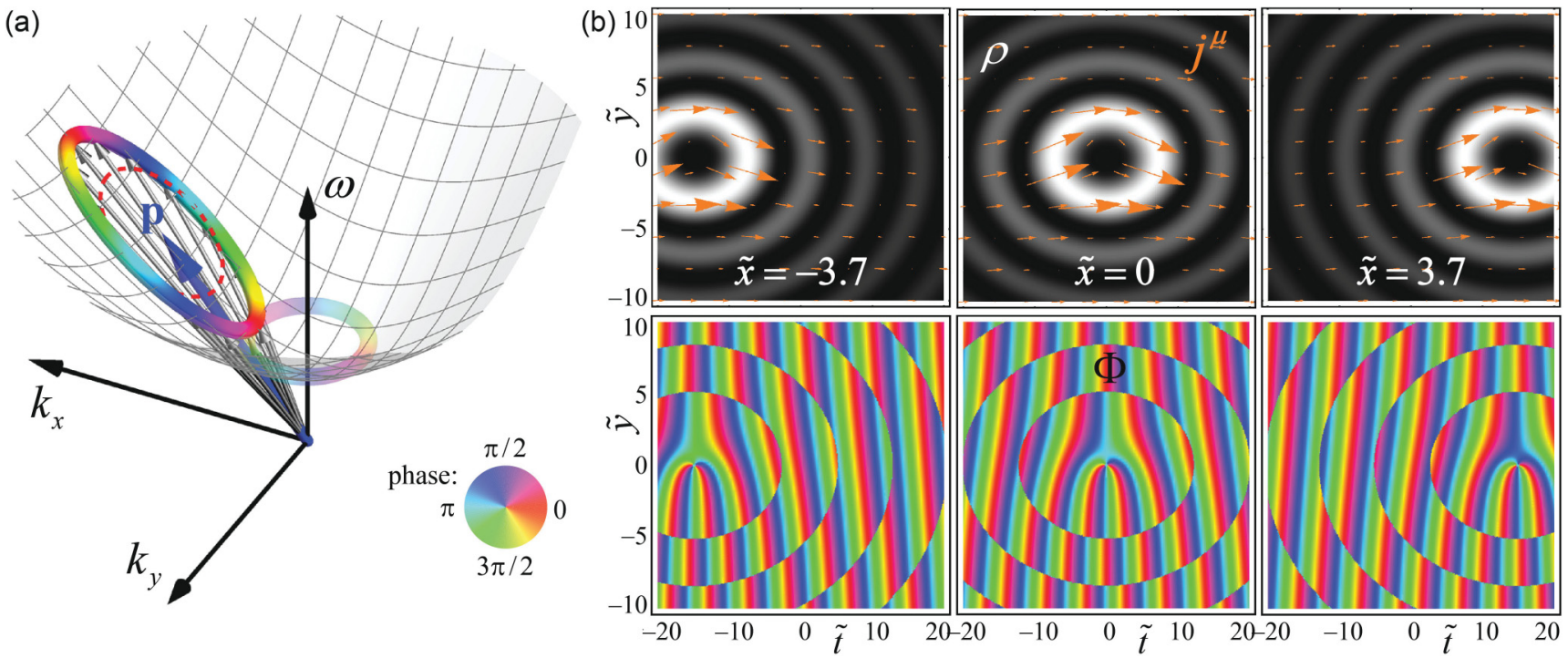

FIG. 5. (Color online) Spatiotemporal representation of the transversely moving Bessel beam from Fig. 4. (a) Here its spectrum is plotted on the mass hyperboloid in the $\left(\omega, k_{x}, k_{y}\right)$ space where it is seen that it also forms an elliptical vortex loop if projected onto the $\left(\omega, k_{y}\right)$ plane. (b) The real-space evolution of the beam is shown here in the form of the density, current, and phase distributions in the $(t, y)$ plane for different values of $x$ [cf. Fig. 4(b)]. The corresponding components of the four-current $j^{\mu}$ are $\left(j^{0}, j_{y}\right)$, where $j^{0}=-\operatorname{Im}\left(\psi^{*} \partial_{t} \psi\right)$.

It has the form of a mixed edge-screw dislocation [1,2], i.e., a spatiotemporal vortex in the $(t, x, y)$ space, with its singularity line $\{y=0, x=v t\}$ being parallel to the $z$ axis in space and moving along the $x$ axis with time. Figure 5(b) illustrates the spatiotemporal character of the Lorentz-boosted beam (14) by showing its evolution in the $(t, y)$ plane for different values of $x$. One can see that the $x$-moving vortex appears in the $(t, y)$ plane entirely similar to the $t$-moving vortex in the $(x, y)$ plane.

Similar to the example discussed in the previous section, the transverse current and motion of the Lorentz-boosted beam implies a tilted momentum of the field, $p_{x} \neq 0$, whereas the OAM is still determined by the $z$ direction of the vortex axis. Calculating the expectation values, Eqs. (6) and (7), and assuming paraxiality of the beam, $\gamma k_{\perp 0} \ll \sqrt{k_{z 0}^{2}+\left(\gamma v \omega_{0}\right)^{2}}$, we obtain

$$
\begin{aligned}
& E \simeq \omega_{c}=\sqrt{\omega_{0}^{2}+\left(\gamma v \omega_{0}\right)^{2}}=\gamma \omega_{0}, \quad \mathbf{R}_{\perp} \simeq v t \mathbf{e}_{x}, \\
& \mathbf{p} \simeq k_{z 0} \mathbf{e}_{z}+\gamma v \omega_{0} \mathbf{e}_{x}, \quad \mathbf{L} \simeq \frac{\gamma+\gamma^{-1}}{2} \ell \mathbf{e}_{z} .
\end{aligned}
$$

Here the values of the transverse coordinates and the momentum correspond to the linear motion of the beam, $d \mathbf{R}_{\perp} / d t=\mathbf{p}_{\perp} / E$, whereas the transformation of the OAM, which acquires the factor $\left(\gamma+\gamma^{-1}\right) / 2$, owes its origin to the elliptical relativistic deformation of the beam $[9,20]$. Indeed, the OAM is given by the cross product of the position and the momentum, $\hat{L}_{z}=\hat{x} \hat{p}_{y}-\hat{y} \hat{p}_{x}$, and both summands $\hat{x} \hat{p}_{y}$ and $-\hat{y} \hat{p}_{x}$ provide equal contributions of $L_{z} / 2$ to the intrinsic OAM of the cylindrical beam (5). In the transversely moving beam, Eqs. (14) and (15), the Lorentz transformation results in the contraction of the coordinate $x$ and expansion of the momentum component $k_{x}$ (Fig. 4), and the above two summands acquire factors $\gamma^{-1}$ and $\gamma$, respectively. Thus, Eqs. (12)-(18) and Figs. 4 and 5 demonstrate the existence of spatiotemporal nondiffracting Bessel beams with noncollinear momentum and intrinsic OAM, L $\Varangle \mathbf{p}$. Note that the collinearity of $\mathbf{p}$ and $\mathbf{L}$ is broken upon the Lorentz transformation because the momentum is transformed as a vector, while the OAM is transformed as a part of the antisymmetric angular-momentum tensor [9].

\section{RELATIVISTIC HALL EFFECT}

It should be noticed that the last equation (18) contradicts the Lorentz transformation of the angular momentum $\mathbf{L}$ of a point particle. Indeed, the Lorentz transformation of the original OAM $\mathbf{L}=\ell \mathbf{e}_{z}$ should yield $\mathbf{L}^{\prime}=\gamma \ell \mathbf{e}_{z}$ after the boost (13). This apparent paradox is considered in detail in Ref. [9]. It is resolved when one takes into account the "relativistic Hall effect," i.e., the transverse $y$ deformation and shift of the OAM-carrying object observed in an $x$-moving frame. It turns out that the geometric center of such an object undergoes a transverse shift of its centroid:

$$
Y \simeq-\frac{v}{2 E_{0}} L=-\frac{v \ell}{2 \omega_{0}} .
$$

Together with the momentum component $p_{x} \simeq \gamma v \omega_{0}$, Eq. (18), the shift (19) generates extrinsic $O A M L^{\text {(ext) }}=$ $-Y p_{x}=\gamma v^{2} \ell / 2$. Adding it to the intrinsic OAM (18), we obtain

$$
L^{(\mathrm{int})}+L^{(\mathrm{ext})} \simeq \frac{\gamma+\gamma^{-1}}{2} \ell+\frac{\gamma v^{2}}{2} \ell=\gamma \ell,
$$

in agreement with the Lorentz transformation.

We did not observe the shift (19) in Figs. 4 and 5, because we used the "naive" density $\rho=|\psi|^{2}$ and the current $\mathbf{j}=\operatorname{Im}\left(\psi^{*} \nabla \psi\right)$. Indeed, $\rho$ does not describe the density of particles in the case of the relativistic Klein-Gordon equation (1) because the volume integral $\int \rho d V$ is not Lorentz invariant. The proper particle density and current are given by [18]

$$
\rho_{P}=-\operatorname{Im}\left(\psi^{*} \partial_{t} \psi\right), \quad \mathbf{j}_{P}=\operatorname{Im}\left(\psi^{*} \nabla \psi\right),
$$



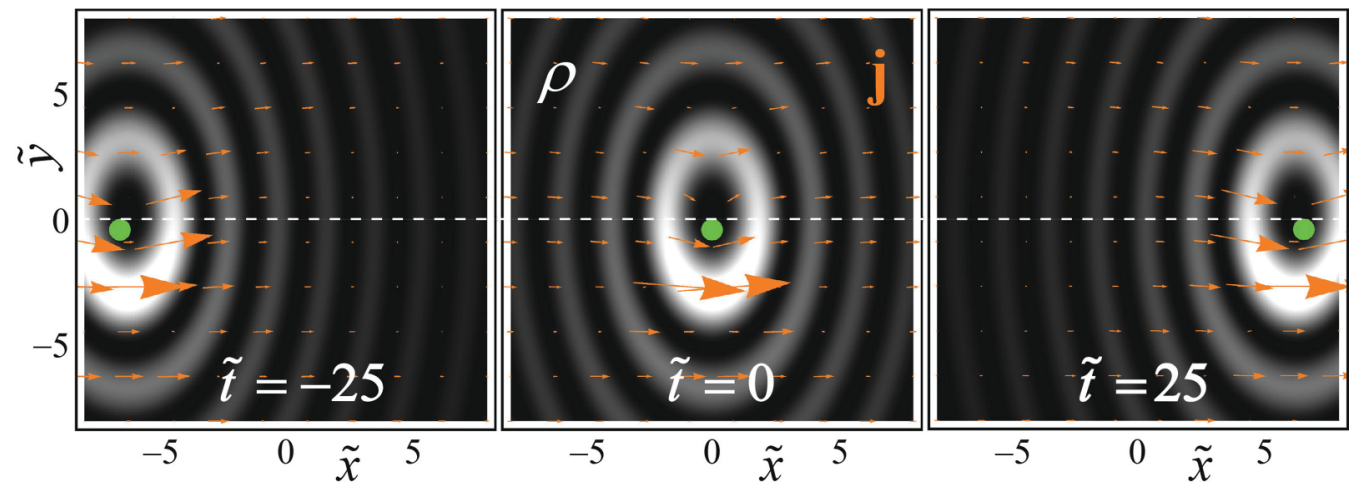

FIG. 6. (Color online) Same evolution of the Lorentz-boosted Bessel beam (14) as in Fig. 4, but here with the scaling $\tilde{\psi} \rightarrow \sqrt{\omega} \tilde{\psi}$ of the spectral Fourier amplitudes. Thus, $\rho=|\psi|^{2}$ becomes the true relativistic probability density, and $\mathbf{j}=\operatorname{Im}\left(\psi^{*} \nabla \psi\right)$ becomes the energy current (momentum density). This causes the relativistic Hall effect [9]: a tiny $y$ deformation and subwavelength shift (19) of the beam which is seen here owing to the nonparaxial character (small radius) of the beam. The dot indicates the center of gravity of the beam according to Eqs. (18) and (19): $\mathbf{R}_{\perp} \simeq\left(v t,-v \ell / 2 \omega_{0}\right)$, and the small $x$ deviations of the dot are due to the nonparaxial corrections.

whereas the energy density and the current (momentum density) are

$$
\begin{aligned}
\rho_{E} & =\frac{1}{2}\left[\left|\partial_{t} \psi\right|^{2}+|\nabla \psi|^{2}+m^{2}|\psi|^{2}\right], \\
\mathbf{j}_{E} & =-\operatorname{Re}\left[\left(\partial_{t} \psi\right)^{*}(\nabla \psi)\right] .
\end{aligned}
$$

For plane waves, this yields simple $\omega$ scalings: $\rho_{E}=\omega^{2} \rho$, $\mathbf{j}_{E}=\omega \mathbf{j}$, and $\rho_{P}=\omega \rho$, which make no difference for monochromatic beams in the rest frame. However, a Lorentz transformation to the moving frame affects these distributions via local variations of the frequency. In other words, one can use the naive density $\rho$ and the current $\mathbf{j}$, instead of $\rho_{P}$ and $\mathbf{j}_{\mathbf{E}}$ which should be used in Eqs. (6), but the amplitudes of the Fourier spectral components should be scaled as $\tilde{\psi} \rightarrow \sqrt{\omega} \tilde{\psi}$.

In Fig. 6 we plot the spatiotemporal evolution of the Lorentz-boosted beam with the Fourier amplitude scaling $\tilde{\psi} \rightarrow \sqrt{\omega} \tilde{\psi}$ taking into account the relativistic Hall effect [9]. The $y$ deformation and shift (19) are clearly seen due to the chosen nonparaxial relativistic parameters of the beam. However, for paraxial or nonrelativistic beams the tiny shift (19) (less than a fraction of the wavelength) is practically imperceptible. Moreover, we emphasize that both the spatiotemporal beams with scaling $\tilde{\psi} \rightarrow \sqrt{\omega} \tilde{\psi}$ and those without it are exact solutions of the Klein-Gordon wave equation and both can exist.

\section{CONCLUDING REMARKS}

We have demonstrated the existence of spatiotemporal vortex wave beams in free space. Such beams move uniformly in the transverse direction and carry intrinsic orbital angular momentum noncollinear to their mean momentum. Furthermore, the spatiotemporal vortex around the beam axis appears as a moving edge-screw dislocation in both the spatial and the space-time cross sections of the beam. The spatiotemporal vortex beams are polychromatic and can naturally appear in problems with nonstationary (moving) sources or media. Localized wave packets with spatiotemporal vortices and tilted intrinsic OAM can readily be constructed by considering slightly delocalized (e.g., Gaussian) Fourier spectra instead of the $\delta$ functions considered here. It should be emphasized that our analysis is valid for both massless and massive fields, in both relativistic and nonrelativistic cases.

Quite naturally, a complete relativistic family of spatiotemporal Bessel beams is constructed via the Lorentz transformations of the spatial (monochromatic) Bessel beams. This means that even the stationary OAM states of light or quantum particles will be seen as spatiotemporal states in the case of a transversely moving observer or source. In this manner, the deformation of the phase pattern of a moving vortex (cf. Figs. 2 or 4 with Fig. 1) offers a sensitive interferometric tool detecting relativistic effects even at nonrelativistic velocities. Indeed, purely relativistic deformations of the phase fronts (intimately related to the Lorentz transformation of time [9]) become significant for velocities $v$ comparable with $c^{2} / \omega r \sim$ $c^{2} k_{\perp 0} / \omega$, where $r \sim k_{\perp 0}^{-1}$ is the beam radius. For paraxial beams with $\theta \equiv k_{\perp 0} c / \omega \ll 1$, this allows the observation of strong deformations at speeds $v \sim \theta c \ll c$. This is explained by the fact that the transverse phase velocity of the motion of the phase fronts is much larger than the speed of light, $\omega / k_{\perp 0}=\theta^{-1} c \gg c$.

Spatiotemporal OAM states of light or particles can appear in a variety of systems involving moving frames and sources. In particular, they can be created upon scattering by moving objects, emitted by satellites doing quantum communications [11] or by natural astrophysical sources [13,14], and produced in collisions of high-energy particles [12]. Furthermore, transversely moving vortex solutions can be important in twodimensional wave fields, such as surface plasmon polaritons [21]. While we have discussed the simplest vortex-beam configurations, belonging to the Bessel-beam family, note that optical vortex lattices can appear from the generic interference of only three monochromatic plane waves [22]. In a similar manner, an infinite lattice of spatiotemporal vortices can appear from the generic interference of three plane waves with different wave vectors and frequencies. This offers a simple way of generating spatiotemporal vortices in an optical laboratory.

In this work we only considered scalar waves and orbital angular momentum. For vector (e.g., electromagnetic) waves carrying spin angular momentum, the relativistic 
transformation properties can be more complicated. Indeed, the spin angular momentum is produced by a circulating spin current, which, however, does not transport energy [17,23]. This current is unrelated to phase gradients and has specific properties distinct from those of the regular orbital current. Although one could expect that the integral angular momentum of the beam is transformed in the same way independently of its spin or orbital nature, the internal deformations of the beam can be different. For instance, it is known that spin has a purely intrinsic nature and cannot have extrinsic contributions produced by the relativistic Hall effect, Eq. (20). Relativistic transformations of vector beams carrying both spin and orbital angular momenta will be considered in detail elsewhere.

\section{ACKNOWLEDGMENTS}

We acknowledge fruitful discussions with I. P. Ivanov, correspondence with A. P. Sukhorukov, and support from the European Commission (Marie Curie Action), ARO, JSPSRFBR Contract No. 12-02-92100, Grant-in-Aid for Scientific Research (S), MEXT Kakenhi on Quantum Cybernetics, and the JSPS via its FIRST program.
[1] J. F. Nye and M. V. Berry, Proc. R. Soc. London A 336, 165 (1974).

[2] M. V. Berry, in Les Houches Lecture Series Session XXXV, edited by R. Balian, M. Kléman, and J-P. Poirier (North-Holland, Amsterdam, 1981); J. F. Nye, Natural Focusing and Fine Structure of Light (IOP Publishing, Bristol, 1999); M. S. Soskin and M. V. Vasnetsov, Prog. Opt. 42, 219 (2001).

[3] L. Allen, M. W. Beijersbergen, R. J. C. Spreeuw, and J. P. Woerdman, Phys. Rev. A 45, 8185 (1992).

[4] L. Allen, M. J. Padgett, and M. Babiker, Prog. Opt. 39, 291 (1999); L. Allen, S. M. Barnett, and M. J. Padgett, Optical Angular Momentum (Taylor \& Francis, London, 2003); S. Franke-Arnold, L. Allen, and M. J. Padgett, Laser Photon. Rev. 2, 299 (2008); J. P. Torres and L. Torner, Twisted Photons (Wiley-VCH, New York, 2011).

[5] K. Y. Bliokh, Y. P. Bliokh, S. Savel'ev, and F. Nori, Phys. Rev. Lett. 99, 190404 (2007); K. Y. Bliokh, M. R. Dennis, and F. Nori, ibid. 107, 174802 (2011).

[6] M. Uchida and A. Tonomura, Nature (London) 464, 737 (2010); J. Verbeek, H. Tian, and P. Schattschneider, ibid. 467, 301 (2010); B. J. McMorran et al., Science 331, 192 (2011); J. Verbeek et al., Appl. Phys. Lett. 99, 203109 (2011).

[7] B. T. Hefner and P. L. Marston, J. Acoust. Soc. Am. 106, 3313 (1999); C. E. M. Demore, Z. Yang, A. Volovick, S. Cochran, M. P. MacDonald, and G. C. Spalding, Phys. Rev. Lett. 108, 194301 (2012).

[8] A. P. Sukhorukov and V. V. Yangirova, Proc. SPIE 5949, 594906 (2005); A. K. Satarin, A. P. Sukhorukov, and V. V. Yangirova, Bull. Russ. Acad. Sci. Phys. 70, 2020 (2006).

[9] K. Y. Bliokh and F. Nori, Phys. Rev. Lett. 108, 120403 (2012).

[10] J. Leach, A. J. Wright, J. B. Gotte, J. M. Girkin, L. Allen, S. Franke-Arnold, S. M. Barnett, and M. J. Padgett, Phys. Rev. Lett. 100, 153902 (2008); S. Franke-Arnold, G. Gibson, R. W. Boyd, and M. J. Padgett, Science 333, 65 (2011).
[11] P. Villoresi et al., New J. Phys. 10, 033038 (2008).

[12] I. P. Ivanov, Phys. Rev. D 83, 093001 (2011); Phys. Rev. A 85, 033813 (2012).

[13] B. Thidé, H. Then, J. Sjoholm, K. Palmer, J. Bergman, T. D. Carozzi, Y. N. Istomin, N. H. Ibragimov, and R. Khamitova, Phys. Rev. Lett. 99, 087701 (2007); F. Tamburini et al., Nat. Phys. 7, 195 (2011).

[14] G. C. G. Berkhout and M. W. Beijersbergen, Phys. Rev. Lett. 101, 100801 (2008).

[15] D. McGloin and K. Dholakia, Contemp. Phys. 46, 15 (2005).

[16] K. Y. Bliokh, M. A. Alonso, E. A. Ostrovskaya, and A. Aiello, Phys. Rev. A 82, 063825 (2010).

[17] M. V. Berry, J. Opt. A: Pure Appl. Opt. 11, 094001 (2009); A. Y. Bekshaev, K. Y. Bliokh, and M. S. Soskin, J. Opt. 13, 053001 (2011).

[18] V. B. Berestetskii, E. M. Lifshitz, and L. P. Pitaevskii, Quantum Electrodynamics (Pergamon, Oxford, 1982).

[19] I. V. Basistiy, V. Y. Bazhenov, M. S. Soskin, and M. V. Vasnetsov, Opt. Commun. 103, 422 (1993); M. R. Dennis, Opt. Lett. 31, 1325 (2006).

[20] V. G. Fedoseyev, J. Phys. A: Math. Gen. 41, 505202 (2008); K. Y. Bliokh, I. V. Shadrivov, and Y. S. Kivshar, Opt. Lett. 34, 389 (2009).

[21] V. E. Lembessis, M. Babiker, and D. L. Andrews, Phys. Rev. A 79, 011806(R) (2009); P. S. Tan et al., Opt. Lett. 36, 3287 (2011); A. P. Sukhorukov, D. O. Ignatyeva, and A. N. Kalish, J. Infrared, Millimeter, Terahz. Waves 32, 1223 (2011).

[22] J. Masajada and B. Dubik, Opt. Commun. 198, 21 (2001); K. O’Holleran, M. J. Padgett, and M. R. Dennis, Opt. Express 14, 3039 (2006).

[23] H. C. Ohaanian, Am. J. Phys. 54, 500 (1986); K. Mita, ibid. 68, 259 (2000). 